\title{
Current experiences with Internet telepathology and possible evolution in the next generation of Internet services
}

\author{
V. Della Mea and C.A. Beltrami * \\ Institute of Pathology, University of Udine, Italy
}

The last five years experience has definitely demonstrated the possible applications of the Internet for telepathology. They may be listed as follows: (a) teleconsultation via multimedia e-mail; (b) teleconsultation via web-based tools; (c) distant education by means of World Wide Web; (d) virtual microscope management through Web and Java interfaces; (e) real-time consultations through Internet-based videoconferencing. Such applications have led to the recognition of some important limits of the Internet, when dealing with telemedicine: (i) no guarantees on the quality of service (QoS); (ii) inadequate security and privacy; (iii) for some countries, low bandwidth and thus low responsiveness for real-time applications. Currently, there are several innovations in the world of the Internet. Different initiatives have been aimed at an amelioration of the Internet protocols, in order to have quality of service, multimedia support, security and other advanced services, together with greater bandwidth.

The forthcoming Internet improvements, although induced by electronic commerce, video on demand, and other commercial needs, are of real interest also for telemedicine, because they solve the limits currently slowing down the use of Internet. When such new services will be available, telepathology applications may switch from research to daily practice in a fast way.

Keywords: Telepathology, Internet, multimedia, dynamic telepathology

\section{Introduction}

Telepathology has been one of the most growing telemedicine fields in the recent years. At the same

\footnotetext{
${ }^{*}$ Corresponding author: Prof. Carlo Alberto Beltrami, Institute of Pathology, University of Udine, p.le S. Maria della Misericordia, 33100 Udine, Italy. Fax: +39 432 559420; E-mail: beltrami@ uniud.it.
}

time, also the Internet use has rapidly grown, thus several research efforts have been put in the use of its protocols for telemedicine tasks, starting from electronic mail and including the World Wide Web, with some attention also on the basic Internet protocols that can be used in dynamic telepathology.

The present paper introduces at first the main telepathology tasks and problems, and then explores some Internet-based approaches ongoing around the world, ranging from the use of multimedia e-mail, to collaboration tools, to web-based interfaces for robotized microscopes. A quick overview on the expected evolution of the Internet in the next few years will provide the framework for future telemedical applications.

\section{Telepathology}

Independently from the aim, the most important source of images for pathologist is surely the glass slide observed through an optical microscope, although other fundamental information is provided by the macroscopical aspect of surgical samples, by electron microscope images, etc., all complemented with patient' data and history. The pathologist observes the glass slide at different objective magnifications, looking for diagnostic clues that can be found in specific optical fields.

So, telepathology is mainly based on the delivery of microscope images, by means of two main approaches, as in other telemedicine fields: real-time [27] and storeand-forward [3] methods. The former is based on the direct dialogue between two entities, and usually needs greater resources than the latter, which is intrinsically asynchronous and furthermore does not need the presence of both interlocutors at the same time.

The choice between store-and-forward and real-time for telepathology does not depend only from the available bandwidth: in order to make store-and-forward 
possible, a sampling of the glass slide should be made by the referring pathologist, by choosing the images regarded as most representative of the case. Those images could then be sent to another pathologist for consultation, or stored in a case archive for education, or even used for quality control of cytopathologic laboratories; however, they represent only a part of the original case, hopefully the most diagnostically or educationally interesting.

On the other side, real-time telepathology allows the recipient pathologist for interactively selecting fields following a variety of approaches, based either on the real-time exchange of static images or on the delivery of real-time video from the microscope; sometimes, in addition to this there is the availability of a robotized microscope, which allows for its full remote movement. In general, effective real-time telepathology needs high bandwidth, and often expensive tools (e.g., robotized microscopes); in addition, videoconferencebased tools have a low image quality, which is not always adequate to diagnostic aims [20].

The above reasons push towards a distinction between tasks that can be carried out using the less powerful but even inexpensive store-and-forward telepathology and those needing the real-time methods. Among the former, remote expert consultation is being greatly studied and discussed, because its store-andforward version is particularly attractive as a method for international referral, whereas real-time telepathology is the right method for the intraoperative diagnosis, which has very strict time constraints and the need of completely observing a specimen.

As it can be seen in the following, store-and-forward communications can be easily realised through Internet protocols; real-time too, in principle, but with quality problems due to the absence of guarantees on performance.

\section{The Internet}

The evolution of the Internet derives by a common effort on protocol studies, proposals and implementations. Protocols, either already accepted as standard, as well as proposals and Internet committee drafts are described in publicly available documents, which are released on the Internet itself.

Internet protocols are based on the client/server paradigm, which is a computational architecture that involves client processes requesting services from server processes. The communication between client and server is carried out according to a set of precise rules called client-server protocol.

The Internet protocols are organised into a fourlayer stack: the first manages the physical media where the connection is established (e.g., Ethernet), the second (IP, Internet Protocol) allows the exchange of basic data packets, then there is the TCP (Transmission Control Protocol) that provide reliable, connectionoriented, full-duplex streams. Finally there is an application layer that implements some high level service such as electronic mail, World Wide Web, file transfer and so on: this is the layer commonly available to the user programs.

On the Internet, each computer is designated by a 32-bit number, usually written as four numbers separated by dots (e.g., 158.110.1.3). To this number may also be associated a name, composed by labels separated by dots (e.g., hydrus.cc.uniud.it) through the DNS (Domain Name System) protocol.

Security and privacy are crucial needs of telemedicine applications: in its basic form, the Internet does not provide for them, although there exists now an important security enhancement (Secure Socket Layer, SSL). The SSL protocol includes provisions for server authentication (verifying the server's identity to the client), encryption of data in transit, and optional client authentication (verifying the client's identity to the server). Several commercial implementations of SSL are currently available, mainly on WWW servers and browsers, although its use can be wider.

Apart from SSL, multimedia email provides for privacy-enhanced communications basing on public key cryptography and digital signature.

Another form of data protection is given by network firewalls and proxies. A firewall is a method of protecting one network from another non-trusted network, with two main mechanisms: one for traffic blockage and one for traffic permission. In addition, the firewall can be used to trace in-going and out-going information.

Proxies are high-level applications that act as a gateway between specific applications and the network; this allows to filter and $\log$ traffic.

Another important issue for telemedicine is the quality of service. The Internet is born from a military effort, with the aim of providing redundant communications, thus, at least from this point of view, some quality is available; however, there isn't a specific guarantee policy on performance, thus no strictly reliable communications may be made. 


\section{Telepathology on the Internet}

Although there are many research and commercial tools for telepathology, using mainly ISDN (Integrated Services Digital Network) and traditional analogue telephone lines, in the recent years great attention has been posed on Internet-based tools and methods, which attempt to replicate almost all kind of systems, either static or dynamic $[17,19]$.

The Internet protocols and tools considered for implementing telepathology are those mostly used in the current communications, i.e., electronic mail and webrelated methods, but also directly low-level connections, used in dynamic approaches.

In the following, a brief overview of current Internet telepathology application will be given.

\section{Telepathology through electronic mail}

In particular, multimedia electronic mail has been studied since 1995 by the Institute of Pathology of the University of Udine, Italy, together with the research partners of the IRST (Istituto per la Ricerca Scientifica e Tecnologica) and the City Hospital of Trento, Italy, as a way for doing remote expert consultation instead of glass slide mailing [4]. The study involved some initial interoperability problem (now resolved by the most recent mail agents), the analysis of the image sampling issue and the use of the JPEG compression method for reducing storage and transmission time. Several experiments in different pathology fields (including GI, breast cytology, frozen sections, skin pathology) have been carried out since then, demonstrating an overall feasibility, although some problem appears when dealing with specific fields or particularly difficult cases [5-7]. However, email protocols seem to be particularly suited for telemedicine, because they directly provide solutions for many of the telemedical needs: multimedia, ease of use, privacy and security. Other evaluations of the same type has been carried out later, by different research groups, aiming at evaluating the diagnostic accuracy of the method, as well as its privacy features $[2,9,26]$.

As a consequence, several telepathology systems providers (e.g., Olympus, Leica) included into their instruments also a module for doing email consultations. Furthermore, generic tools for store-and-forward telemedicine, including telepathology, started to appear; one of the first - DORIS - has been developed by the Dept. of Telemedicine, University Hospital of Troms $\varnothing$, Norway, in collaboration with NORUT IT.

A way to override the image sampling problem is that of including the whole diagnostic field into an email message. This has been experimented in the specific case of biopsies, which have a very small area [28]. The proposed approach is based on the use of a very high resolution acquisition device known as photoscanner, which applies the technology currently used in flatbed scanners to the acquisition of images. Images can be grabbed from the microscope at a low magnification, but with a resolution such that is as having acquired much more images at a higher magnification, and including the whole biopsy.

\section{Web-based telepathology tools}

On the World Wide Web, telepathology can be carried out by means of servers, where histopathologic cases may be put with different aims, including diagnosis, education and quality control.

The photoscanner previously cited has been proposed also as a way for realising the so-called "virtual microscopy" for teaching use [10,17-20,29]: being possible to represent the whole biopsy in a single image, this could be used as a basis for constructing a virtual glass slide (together with higher magnification images), which can be freely navigated by students and/or specialists doing continuing education, exactly as they were at the microscope. In addition, such an approach has its practical application through the Internet, by means of a web-based interface that makes world-wide accessible such an educational resource. More recently new ways of doing almost the same task have been proposed, i.e., acquiring whole specimens for virtual microscopy. In particular, the Bacus BLISS workstation allows to digitise and send through the Internet a specimen - called "WebSlideC)" for diagnostic as well as educational aims. A WebSlide is composed by a slide overview, created from a patchwork of lowmagnification images, and a number of high resolution images, covering the most interesting specimens areas (eventually the whole slide). Such an image structure can be viewed by means of a specific browser, which simulates the behaviour of the microscope, allowing stage movements and magnification changes.

There are different ways for making such large datasets available on the Internet. Among those, wavelets-based compression algorithms should be cited, because they easily allow the multiresolution display of 
large images without transferring the entire file, and the FlashPix format, with almost the same features.

From Japan comes an approach to diagnostic telepathology, which allows also for interactivity. Nagata et al. [25] (National Cancer Center Research Institute, Tokyo, Japan) propose a whiteboard-like system implemented using the Java language, which is thus independent from the hardware/software platform adopted. The system allows for sharing still images onto a socalled whiteboard, having at disposal some collaboration tool typical of such kind of systems (shared cursor, drawing tools, text areas), all through a standard Web browser. Such an approach is the same as many lowend commercial systems, but instead of compelling interlocutors to have the same kind of hardware and software, it can be used everywhere, making it much more interesting from the point of view of a wide diffusion.

In the USA, AFIP (American Forces Institute of Pathology) is using telepathology since many years for doing international consultations [17]. Among the different methods adopted, there are also Internet-based case submissions. They started with an ftp-based approach, where referring pathologist transferred images into an ftp server; then, in 1996 multimedia email was also introduced. While maintaining the former options, recently they prepared a completely renewed tool for uploading pathologic cases through a secure Web interface, and also an archive of cases derived from consultations, which is of very high value for continuing education being composed by cases coming from around the world (http://www.afip.org/telepathology).

Something similar is arising in Europe from an European Community-funded project called Europath (ended in 1998): from remote consultation to continuing education and quality control, the Internet will be used as a medium for enhancing collaboration among European pathologists thanks to the PathConsult initiative (http://pathconsult.imag.fr/).

A recent and important support to Internet telepathology comes from the UICC (Union Internationale Contre le Cancer), which launched a web-based consultation site to be used for second-opinion diagnosis in pathology [8].

In Italy, three different initiatives have been recently introduced. The Institute of Pathology of Udine is leading a national network of telepathology for research, education and quality control, which bases part of their work on web-based tools (in particular for educational cases and for remote consultation); the pathologists of the Milan area are publishing their slide seminars cases on the Internet; and the Italian Society of Pathology (SIAPEC) has just started an archive for consultations.
A particular telepathology server-based application has been developed by the Euroquant project (http: //euroquant.med.tu-dresden.de/) for support in remote quantitation of DNA cytometry data [10], with the overall aim of helping in the standardisation of such measurements [23], and of promoting uniform quality control criteria [15]. Remote quantitation through the Internet has also been tried using multimedia email.

\section{Dynamic telepathology through the Internet}

Some researchers push even forward the use of Internet, by studying methods and implementing tools for the remote control of microscopes. Although there already existed tools for controlling generic image sources on the Web (e.g., motorised videocameras), pioneer work on this topic has been carried out at the Charite Hospital in Berlin, Germany [32,33], where a Web-based interface to a robotized microscope has been implemented and successfully tested (http://amba.charite.de/telemic/index.html). Again, Java has been used to produce a tool independent of specialised hardware and software. A similar experience has been carried out also in a collateral field by the National Center for Microscopy and Imaging Research, La Jolla, CA, where a web interface to an electron microscope has been realised with the same kind of instruments as above [13].

As a matter of fact, some of the currently available dynamic telepathology tools use the Internet basic protocols over ISDN lines, creating this way a very small Intranet composed by two nodes, which could virtually be transferred on the Internet, if its performance would be adequate.

\section{Educational case archives}

Multimedia case archives are usually considered as pertaining to telepathology, because they allow for the distant sharing of medical data. Of course, the Internet and the Web play a major role in the creation of remotely accessible and possibly distributed archives, and thus there are a lot of attempts to create such kind of applications [10,12,22,24]. Among those, it is worth to cite the efforts towards interoperability and standardisation [1,21], which are of great interest for telemedicine: thus HTML with some extension and DICOM (born for teleradiology) have been experimented as effective ways of sharing cases, together with a variety of coding for diagnostic data. Table 1 proposes a set of addresses of educationally relevant pathology sites. 
Table 1

Multimedia case archives and virtual microscopes

\begin{tabular}{|c|c|}
\hline Site & Address \\
\hline AFIP & http://www.afip.org/homes/bethetele/index.html \\
\hline IOS Press telepathology & http://www.iospress.nl/telepathology/ \\
\hline Virtual Autopsy 2 & http://www.le.ac.uk/pathology/teach/va2/ \\
\hline JHAR - John Hopkins & http://www.netautopsy.org/ \\
\hline \multicolumn{2}{|l|}{ Autopsy Resource } \\
\hline $\begin{array}{l}\text { University of Pittsburgh } \\
\text { Case of the Month }\end{array}$ & http://path.upmc.edu/casemonth.html \\
\hline SLUCare Cases & http://www.slucare.edu/clinical/pathlab/case/ \\
\hline Soc. Ultrastructural & http://sup.ultrakohl.com/cotm.html \\
\hline \multicolumn{2}{|l|}{ Pathology } \\
\hline \multicolumn{2}{|l|}{ Microscopia elettronica } \\
\hline University of Virginia & http://www.med.virginia.edu/medicine/clinical/pathology/CaseStudies \\
\hline Case studies & /CaseStudiesHome.html \\
\hline WEBPATH & http://www-medlib.med.utah.edu/WebPath/LABS/LABMENU.html \\
\hline \multicolumn{2}{|l|}{ Laboratory exercises } \\
\hline Italian Network of telepathology & http://www.telemed.uniud.it/ritap/ \\
\hline Cases of the Milan pathologists & http://www.patologi-milano.org \\
\hline SIAPEC Seminars & http://www.siapec.it/seminari/ \\
\hline Virtual micro & http://gynonc.path.med.umich.edu/GynImaging/testmicro1.htm \\
\hline UPMC Virtual & http://telepathology.upmc.edu/website/cytology1.html \\
\hline \multicolumn{2}{|l|}{ Telepathology } \\
\hline $\begin{array}{l}\text { AmeriPath case of the } \\
\text { month }\end{array}$ & http://www.ameripath.com/teaser.html \\
\hline
\end{tabular}

\section{Future evolutions of the Internet and telepathology}

Currently, there are several innovations in the world of the Internet. Different initiatives have been aimed at an amelioration of the Internet protocols, in order to have quality of service, multimedia support, security and other advanced services, together with greater bandwidth. Among these projects, Internet 2 and NGI (Center for Next Generation Internet) in the USA and TEN-155 in Europe should be mentioned.

The forthcoming Internet improvements, although induced by electronic commerce, video on demand, and other commercial needs, are of real interest also for telemedicine, because they solve the limits currently slowing down the use of Internet. When such new services will be available, telepathology applications may switch from research to daily practice in a fast way, although this will probably be possible only in the developed countries.

Among the problems that currently restrain the deployment of the Internet in telemedicine, the most significant is surely the low and unstable performance. However, in the future of the Internet there will be evo- lutions towards an increase of the speed and some form of guarantee on the performance, as explained in the following paragraphs.

\section{Next generation Internet}

IPv6 - or IPng - is a new version of the basic Internet protocols, which introduces a number of enhancements to the current version, known as IPv4. One of the main motivations for its introduction is the shortage of addresses with the current address space.

IPv6 is designed to run on high performance networks as well as on low bandwidth networks, such as wireless communications. In addition, it embodies capabilities needed for some new services available in the near future:

- addressing and routing issues,

- a larger address space (128 bit addresses),

- auto-configuration capabilities,

- simplified IP packets,

- native security,

- multicast and anycast features, 
- "quality of service" functions (through message priority extensions, and resource reservation protocols).

These improvements will give to Internet a greater suitability for telemedicine applications, mainly thanks to security and quality of service features.

In particular, the availability of secure transaction methods will certainly make easier the transfer of telemedicine from research to clinical practice. Some further development is foreseen for Internet real time communications through specialised protocols, i.e., RTP (Real-time Transmission Protocol), which will be of great interest for image and video transmission.

As a practical example of the effect of next generation Internet on the practice of telepathology, let's consider a intra-operative telepathology session to be held during a surgical operation. First of all, the hospital hosting the intervention may reserve the bandwidth needed for a real-time, dynamic connection (e.g., $512 \mathrm{kbit} / \mathrm{s}$ ) for the time of the intervention, between its network and the site of the consultant, or of the consultants, if more than one. When the surgical sample is ready and the glass slide is prepared, the connection is activated having the requested bandwidth available and guaranteed, and, if the opinion of different pathologists is needed, the multicast feature is used in order to connect to them at the same time. During the session, communications occur in a secure and private way, by means of the security features embedded in the protocols, and thus they satisfy the usual legal constraints on safety of patient' data.

\section{Faster communications and telepathology}

In addition to the above evolutions, there will be certainly a growth of available bandwidth, which is needed to support the ever increasing Internet traffic. In particular, the European research networks will take advantage from the TEN-155 project, aimed at bringing $155 \mathrm{Mbit} / \mathrm{s}$ communications and managed bandwidth capabilities among the European countries; similar efforts are ongoing also in the US. At the physical level ATM (Asynchronous Transfer Mode) is regarded as a choice for the implementation of network backbones, while ADSL (Asymmetric Digital Subscriber Line) allows to reach almost every site with a sufficiently high bandwidth. At the present time and likely in the near future, ISDN remains an appropriate choice for intermittently connected telepathology workstations, such as those needed for intraoperative telediagnosis; this also thanks to its guarantees on the quality of service and fixed bandwidth. However, unfortunately such kind of lines will be available firstly in developed countries, increasing the gap known as "digital divide" even in telemedical applications [30]. As an example, actually only 7 out of 54 African countries have ISDN connectivity, 16 countries have Internet access nation-wide, an estimated number of 25000 30000 hosts is permanently connected to the Internet, and there are about 1 million of Internet users but 650000 are located in South Africa [16]. So, although there are a number of collaborative initiatives between developed and developing countries in the field of telemedicine, the technological differences still suggest to adopt low-cost solutions [11].

Increased bandwidth may become also a viable solution to the image sampling problem in static telepathology. The scanning of the whole specimen at different magnifications can already be made, but results in hundreds of megabytes of needed storage. Such "digital glass slides" can be of very high value for education, due to the possibility of simulating the behaviour of a microscope, but if their transmission could be made fast, they may become also a good way of doing telediagnosis without any limitation. However, even "classical" dynamic telepathology will take advantage from higher transmission speeds, being natively more dependent on bandwidth.

\section{Discussion}

Technically, the Internet seems adequate for telepathology applications, although it lacks of quality of service guarantees; protocols are suitable for implementing a wide range of solutions, and already available tools can be applied directly for implementation. In particular, Internet protocols partially override the main problem of every communication-based activity: interoperability and standards.

A crucial problem is that not everywhere the Internet has the same performance. From this point of view, store-and-forward telepathology could be the best solution, although some concern on image sampling still exists. Furthermore, email with its easy-to-use privacy enhancements has been also considered as the future of telemedicine, as reported at MEDNET98 by Ferguson and Greene [31], thus it should be seriously evaluated as a practical mean for exchanging telemedical information. 
However, Internet applications including telepathology can be easily exploited on private intranets, where performance and security are higher than outside, having thus the advantage of open standards without renouncing to speed and privacy. Performance and security will be also guaranteed by the next generation Internet protocols, allowing thus the use of the Internet for real world telepathology applications. This could help in solving at least one of the residual problems described by Wells and Sowter [31], i.e., the data transmission speed; however, the effective adoption of advanced telematics tools inside healthcare institutions is dependent on investments, making often unavailable those tools even when technically possible.

\section{Acknowledgements}

This work was partially supported by a grant of MURST (Ministry of the University), project no. 9906348341.

\section{References}

[1] B. Binder, P. Schwarzmann and J. Schmid, Interoperability aspects within a telepathology network, Adv. Clin. Pathol. 2 (1998), 170-173.

[2] C. Clemente, S. Rao and A. Clemente, Acquisition and transmission of images in anatomic pathology: our experience between Internet and ISDN, Adv. Clin. Pathol. 2 (1998), 151.

[3] V. Della Mea, Store-and-forward telepathology, in: European Telemedicine 1998/99, B. Hernandez and R.Wootton, eds, EHTO/RSM Press/Kensington Publications, London, 1999.

[4] V. Della Mea, Telepathology applications of the Internet multimedia electronic mail, Medical Informatics 23 (1998), 237244 .

[5] V. Della Mea, S. Forti, F. Puglisi, P. Bellutta, N. Finato, P. Dalla Palma, F. Mauri and C.A. Beltrami, Telepathology using Internet multimedia electronic mail: remote consultation on gastrointestinal pathology, J. Telemed. Telecare 2 (1996), 28-34.

[6] V. Della Mea, F. Puglisi, S. Bonzanini, S. Forti, V. Amoroso, R. Visentin, P. Dalla Palma and C.A. Beltrami, Fine needle aspiration cytology of the breast: a preliminary report on telepathology through Internet multimedia electronic mail, Modern Pathol. 10 (1997), 636-641.

[7] V. Della Mea, P. Cataldi, S. Boi, N. Finato, P. Dalla Palma and C.A. Beltrami, Image sampling issues in static telepathology for frozen section diagnosis, J. Clin. Pathol. 52 (1999), 761765.

[8] M. Dietel, T.N. Nguyen-Dobinsky and P. Hufnagl, The UICC Telepathology Consultation Center. International union against cancer. A global approach to improving consultation for pathologists in cancer diagnosis, Cancer 89 (2000), 187-191.
[9] V. Eusebi, L. Foschini, S. Erde and J. Rosai, Transcontinental consults in surgical pathology via the Internet, Human Pathol. 28 (1997), 13-16.

[10] C.L. Felten, J.S. Strauss, D.H. Okada and A.M. Marchevsky, Virtual microscopy: high resolution digital photomicrography as a tool for light microscopy simulation, Human Pathol. 30 (1999), 477-483.

[11] H.S. Fraser and S.J. McGrath, Information technology and telemedicine in sub-Saharan Africa, Br. Medic. J. 321 (2000), 465-466.

[12] P. Furness and J. Rashbass, The virtual double-headed microscope: telepathology for all?, Histopathology 36 (2000), 182183

[13] M. Hadida-Hassan, S.J. Young, S.T. Peltier, M. Wong, S. Lamont and M.H. Ellisman, Web-based telemicroscopy, J. Struct. Biol. 125 (1999), 235-245.

[14] G. Haroske, W. Meyer, M. Oberholzer, A. Boecking and K.D. Kunze, Competence on demand in DNA image cytometry, Pathol. Res. Practice 196 (2000), 285-291.

[15] G. Haroske, W. Meyer, D. Kunze and A. Boecking, Quality control measures for DNA image cytometry in a telepathology network, Adv. Clin. Pathol. 2 (1998), 143-145.

[16] M. Jensen, African Internet connectivity, URL: http://www3. sn.apc.org/africa/ (accessed January 2001).

[17] K. Kayser, J. Szymas and R. Weinstein, Telepathology. Telecommunication, Electronic Education and Publication in Pathology, Springer-Verlag, 1999.

[18] K. Kayser, Telepathology in Europe. Its practical use, Arch. Anat. Cytol. Pathol. 43 (1995), 196-199.

[19] K. Kayser and G. Kayser, Basic aspects of and recent development of telepathology in Europe with specific emphasis on quality assurance, J. Anal. Quant. Cytol. Histol. 21 (1999), 319-328.

[20] K. Kayser, G. Kayser and S. Zink, New technical aspects in telepathology, Elec. J. Pathol. Histol. 6(3) (2000), No. 003-04.

[21] J. Klossa, J.C. Cordier, G. Flandrin, C. Got and J. Hemet, A European de facto standard for image folders applied to telepathology and teaching, Int. J. Med. Inform. 48 (1998), 207-216.

[22] J.D. Kronz, M.A. Silberman, W.C. Allsbrook, S.I. Bastacky, R.T. Burks, S.J. Cina, S.E. Mills, J.S. Ross, W.A. Sakr, J.E. Tomaszewski, L.D. True, T.M. Ulbright, M.W. Weinstein, R.K. Yantiss, R.H. Young and J.I. Epstein, Pathology residents use of a Web-based tutorial to improve Gleason grading of prostate carcinoma on needle biopsies, Human Pathol. 31 (2000), 10441050 .

[23] K.D. Kunze, A. Boecking, G. Haroske, K. Kayser, W. Meyer and M. Oberholzer, Remote quantitation in the framework of telepathology, Adv. Clin. Pathol. 2 (1998), 141-143.

[24] A. Landman, Y. Yagi, J. Gilbertson, R. Dawson, A. Marchevsky and M.J. Becich, Prototype web-based continuing medical education using FlashPix images, in: Proceedings of AMIA Symposium, 2000, pp. 462-466.

[25] H. Nagata and H. Mizushima, A remote collaboration system for telemedicine using the Internet, J. Telemed. Telecare 4 (1998), 89-94. 
[26] I. Nordrum, A. Amin, V. Isaksen, M. Johansen and J.A. Ludvigsen, Still image consultations via e-mail in surgical pathology. A study of diagnostic accuracy, Adv. Clin. Pathol. 2 (1998), 154-156.

[27] I. Nordrum, Real-time diagnoses in telepathology, Adv. Clin. Pathol. 2 (1998), 127-131.

[28] R.P. Singson, S. Natarajan, J.K. Greenson and A.M. Marchevsky, Virtual microscopy and the Internet as telepathology consultation tools. A study of gastrointestinal biopsy specimens, Am. J. Clin. Pathol. 111 (1999), 792-795.

[29] J. Szymas and G. Wolf, Real-time microscopy through the Internet, Elec. J. Pathol. Histol. 4(3) (1998), No. 983-07.
[30] United Nations Development Programme, UNPD Chief warns G8 leaders of a widening "digital divide", URL: http://www.undp.org/dpa/frontpagearchive/july00/21july00/index.html (accessed January 2001).

[31] C.A. Wells and C. Sowter, Telepathology: a diagnostic tool for the millennium?, J. Pathol. 191 (2000), 1-7.

[32] G. Wolf, D. Petersen, M. Dietel and I. Petersen, Telemicroscopy via the Internet, Nature 391 (1998), 613-614.

[33] G. Wolf, I. Petersen and M. Dietel, Microscope remote control with an Internet browser, Analyt. Quant. Cytol. Histol. 20 (1998), 127-132. 


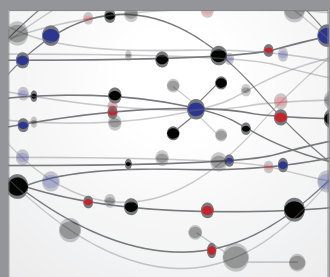

The Scientific World Journal
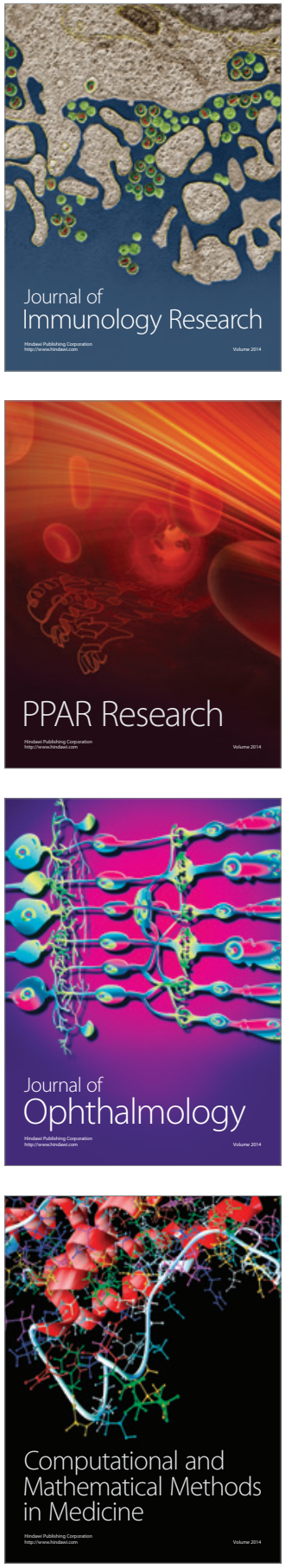

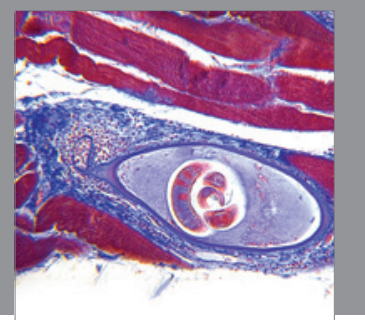

Gastroenterology

Research and Practice
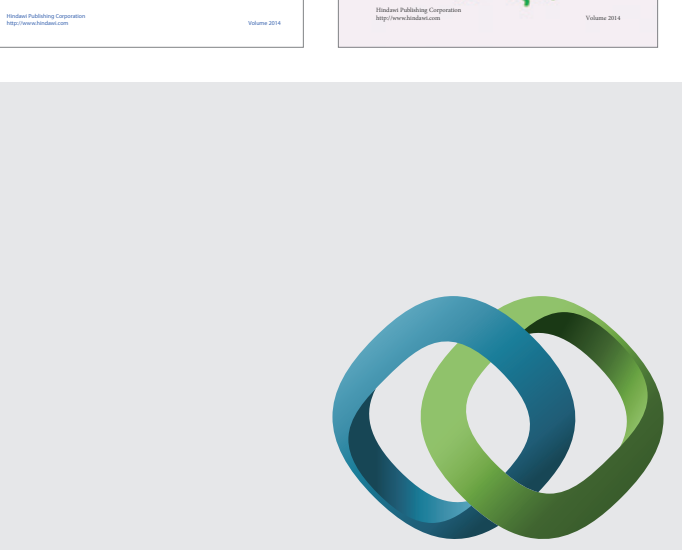

\section{Hindawi}

Submit your manuscripts at

http://www.hindawi.com
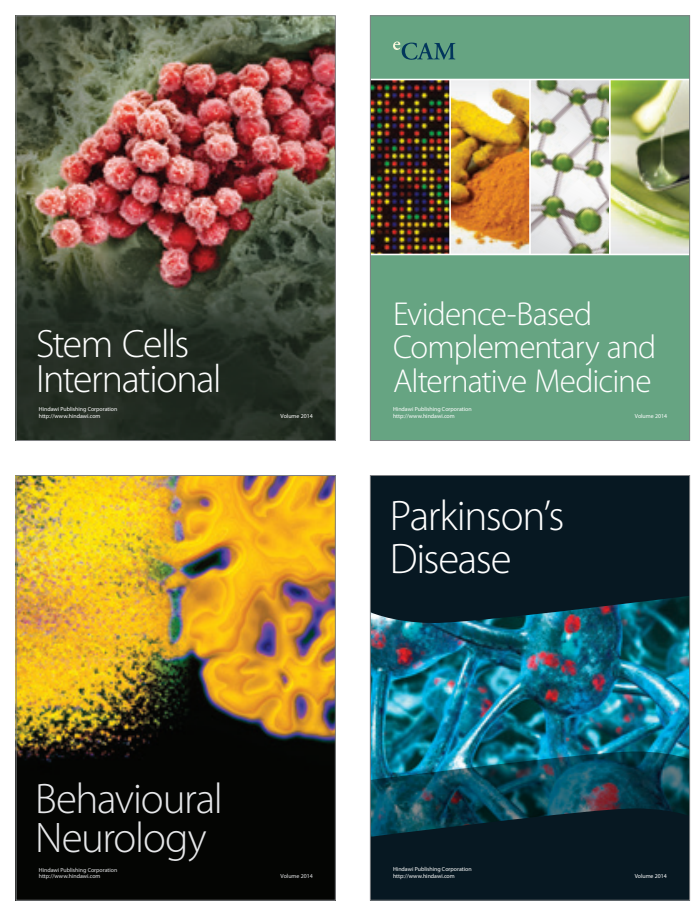

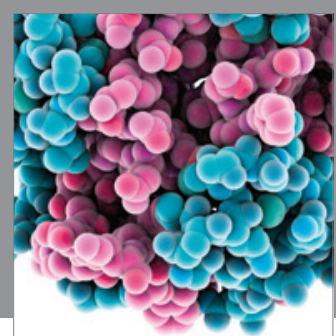

Journal of
Diabetes Research

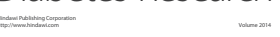

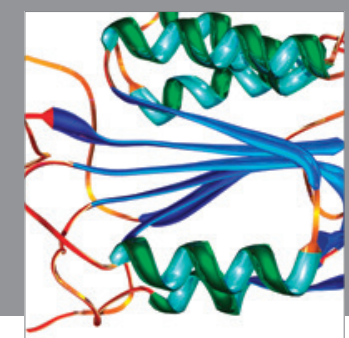

Disease Markers
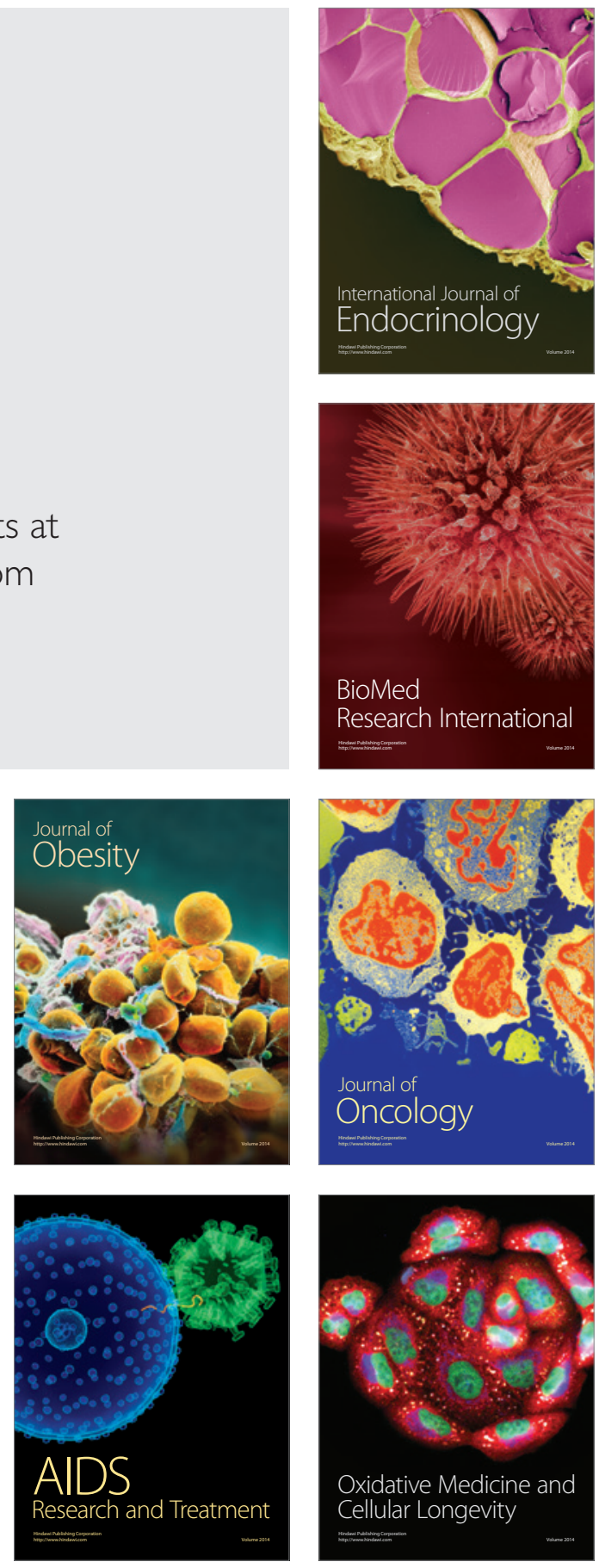